\title{
CONSTITUINTES QUÍMICOS DAS FOLHAS E AVALIAÇÃO DA ATIVIDADE ANTI-INFLAMATÓRIA DE EXTRATOS DAS RAÍZES E FOLHAS DE Guettarda pohliana MÜLL. ARG. (RUBIACEAE)
}

\author{
Gláucio Testa, Paulo Roberto Neves de Oliveira, Cleuza Conceição da Silva, Ivânia Teresinha Albrecht Schuquel e Silvana \\ Maria de Oliveira Santin* \\ Departamento de Química, Universidade Estadual de Maringá, Av. Colombo, 5790, 87020-900 Maringá - PR, Brasil \\ Lucília Kato e Cecília Maria Alves de Oliveira \\ Instituto de Química, Universidade Federal de Goiás, Campus II- Samambaia, CP 131, 74001-970 Goiânia - GO, Brasil \\ Laura Lícia Milani de Arruda e Ciomar Aparecida Bersani-Amado \\ Departamento de Farmacologia e Terapêutica, Universidade Estadual de Maringá, Av. Colombo, 5790, 87020-900 Maringá - PR, \\ Brasil
}

Recebido em 27/5/11; aceito em 12/9/11; publicado na web em 3/11/11

\begin{abstract}
CHEMICAL CONSTITUENTS OF THE LEAVES AND ANTI-INFLAMMATORY ACTIVITY EVALUATION OF EXTRACTS OF ROOTS AND LEAVES OF Guettarda pohliana Müll. Arg. (RUBIACEAE). This phytochemical investigation of Guettarda pohliana leaves led to the isolation of the triterpenes pomolic acid, rotundic acid, $3 \beta, 6 \alpha, 19 \alpha, 23$-tetrahydroxyurs-12-en-28-oic acid, clethric acid, ursolic acid and oleanolic acid, the monoterpenoids loliolide and secoxyloganin, besides daucosterol and steroids. The structures of the isolated compounds were assigned on the basis of NMR data, including two-dimensional NMR methods. The anti-inflammatory activity of the crude methanolic extracts from leaves and roots, as well as of their fractions, was evaluated.
\end{abstract}

Keywords: Guettarda pohliana; triterpenoid; anti-inflammatory activity.

\section{INTRODUÇÃO}

O gênero Guettarda, pertencente à tribo Guettardeae (Rubiaceae), compreende cerca de 150 espécies distribuídas em regiões tropicais e neotropicais. ${ }^{1}$ Espécies do gênero são popularmente utilizadas na América do Sul para tratamento de ferimentos e inflamações. ${ }^{2}$

Guettarda pohliana é uma espécie arbórea conhecida vulgarmente como angélica-do-mato. As espécies brasileiras G. platypoda e $G$. angelica, conhecidas pelo mesmo nome vulgar, são utilizadas popularmente como antifebris e antiespasmódicos ${ }^{3}$ e possuem comprovadas atividades anti-inflamatória ${ }^{3}$ e antibacteriana, ${ }^{4}$ respectivamente.

Em trabalho anterior, realizado com as raízes de Guettarda pohliana, reportou-se o isolamento do ácido quinóvico, de saponinas derivadas dos ácidos quinóvico e cinchólico, do daucosterol e do ácido 4,5-O-dicafeoilquínico, bem como a atividade antioxidante do extrato bruto e de suas frações. ${ }^{5}$

Em continuidade ao estudo da espécie G. pohliana, no presente trabalho descreve-se o isolamento e identificação de seis triterpenos, um monoterpeno, um derivado do ácido clorogênico, um secoiridoide e, ainda, os resultados de atividade anti-inflamatória dos extratos brutos e frações das folhas e das raízes. As estruturas das substâncias isoladas foram definidas com base na análise de dados espectroscópicos de RMN ${ }^{1} \mathrm{H}$ e ${ }^{13} \mathrm{C}$ uni- e bidimensionais (HMQC, HMBC, COSY e NOESY) e a comparação dos dados obtidos com os registrados na literatura.

\section{PARTE EXPERIMENTAL}

\section{Procedimentos experimentais gerais}

Os espectros de RMN (uni- e bidimensionais) foram obtidos em espectrômetro Varian, modelo Mercury plus 300, operando a 300,06 $\mathrm{MHz}$ para ${ }^{1} \mathrm{H}$ e $75,46 \mathrm{MHz}$ para ${ }^{13} \mathrm{C}$. Os deslocamentos químicos são

*e-mail: smoliveira@uem.br dados em ppm, tendo como referência interna o sinal do solvente e/ ou do tetrametilsilano (TMS, $\delta=0,0 \mathrm{ppm}$ ). Para as cromatografias em coluna (CC) utilizou-se gel de sílica 60 (0,063-0,200 mm, Merck) ou Sephadex LH-20, como fase estacionária. Para as cromatografias em camada delgada (CCD) e em camada delgada preparativa (CCDP) empregou-se gel de sílica $60 \mathrm{G}$ e $60 \mathrm{GF}_{254}$ (Merck). A visualização dos compostos em CCD foi realizada por irradiação com luz ultravioleta em 254 e $366 \mathrm{~nm}$ e/ou por pulverização com solução de $\mathrm{H}_{2} \mathrm{SO}_{4} / \mathrm{MeOH}$ (1:1), $\mathrm{H}_{2} \mathrm{SO}_{4}$ /anisaldeído/ácido acético (1:0,5:50 mL) seguido de aquecimento.

\section{Material vegetal}

As folhas da espécie Guettarda pohliana foram coletadas em abril de 2005, às margens da bacia de inundação do alto do Rio Paraná, na região de Porto Rico/PR. A exsicata do material vegetal encontra-se depositada no Herbário da Universidade Estadual de Maringá sob o registro $n^{\circ}$ HUNP 3563.

\section{Isolamento dos constituintes químicos}

As folhas de Guettarda pohliana $(558,0 \mathrm{~g})$ foram secas ao ar, pulverizadas e extraídas exaustivamente com $\mathrm{MeOH}$, à temperatura ambiente, fornecendo 42,1 g de extrato bruto. Parte deste extrato $(23,0 \mathrm{~g})$ foi solubilizado em $\mathrm{MeOH} / \mathrm{H}_{2} \mathrm{O}$ 1:1 e submetido à partição em hexano, $\mathrm{CHCl}_{3}$, AcOEt, o que resultou nas frações hexânica $(1,1$ $\mathrm{g})$, clorofórmica (2,2 g), acetato de etila (2,8 g) e hidrometanólica $(14,9)$. Parte da fração hexânica $(478 \mathrm{mg})$ foi fracionada em gel de sílica com misturas de hexano e AcOEt em ordem crescente de polaridade. A subfração hexano:AcOEt $2 \%(6,0 \mathrm{mg})$ resultante forneceu mistura contendo as substâncias sitosterol, estigmasterol e campesterol. A subfração hexano:AcOEt 15\% (38,0 mg) foi submetida a um novo fracionamento em gel de sílica utilizando como eluentes hexano e AcOEt em gradiente crescente de polaridade, obtendo-se mistura das substâncias $\mathbf{1}$ e 2. Parte da fração clorofórmica (1,51 
g) foi fracionada em gel de sílica utilizando como eluentes hexano, hexano:AcOEt 95-10\% e AcOEt. As subfrações hexano:AcOEt 20\% (37 mg) e hexano:AcOEt 70\% (97,6 mg) resultantes foram submetidas a novo fracionamento em gel de sílica com $\mathrm{CHCl}_{3}$ e AcOEt em ordem crescente de polaridade, resultando no isolamento novamente das substâncias $\mathbf{1}$ (3,0 mg) e $\mathbf{2}$ (3,2 mg) e das substâncias $\mathbf{3}$ e $\mathbf{4}$ como mistura $(7,0 \mathrm{mg})$, respectivamente. As subfrações hexano:AcOEt $50 \%$ e hexano:AcOEt $60 \%$, resultantes do tratamento da fração clorofórmica, apresentaram-se como sólidos amorfos e foram lavados com acetona, resultando na obtenção dos triterpenos 5, 6 e 7 (10 $\mathrm{mg}$ ) como mistura e de daucosterol $(5,0 \mathrm{mg})$, respectivamente. Parte da fração AcOEt (1,2 g) foi submetida a uma filtração em Sephadex LH-20 utilizando-se com eluentes $\mathrm{H}_{2} \mathrm{O} ; \mathrm{H}_{2} \mathrm{O}: \mathrm{MeOH} 25,50$ e $75 \%$ e $\mathrm{MeOH}$ puro, resultando no isolamento de $8(14,8 \mathrm{mg})$ e $9(21,0$ $\mathrm{mg})$. A fração hidrometanólica $(5,0 \mathrm{~g})$ foi dissolvida em $100 \mathrm{~mL}$ de $\mathrm{H}_{2} \mathrm{O} / \mathrm{MeOH}$ 1:1 e submetida à partição em butanol. A fração butanólica resultante $(2,5 \mathrm{~g})$ foi então filtrada em Sephadex LH 20 com $\mathrm{MeOH}$, resultando no isolamento de 9 (46,1 mg). O extrato bruto metanólico das raízes de G. pohliana e as frações deste extrato foram obtidos pelo mesmo procedimento realizado para as folhas. A partir do material seco e pulverizado $(700,1 \mathrm{~g}$ ) foram obtidos $39,1 \mathrm{~g}$ de extrato bruto. $\mathrm{O}$ particionamento deste extrato $(31,0 \mathrm{~g})$ resultou nas frações hexânica (2,6 g), clorofórmica (3,7 g), AcOEt (6,2 g) e hidrometanólica $(17,4 \mathrm{~g})$.

\section{Avaliação da atividade anti-inflamatória}

Empregou-se o modelo de edema de orelha induzido por óleo de cróton em camundongo. ${ }^{6}$ Foram utilizados camundongos Swiss machos e fêmeas, pesando $25 \mathrm{~g}$ em média. $\mathrm{O}$ óleo de cróton foi diluído em uma solução de acetona/água 7:3 e aplicado, em concentração de $200 \mu \mathrm{g} /$ orelha, nas faces internas das duas orelhas dos camundongos. Uma solução $(20 \mu \mathrm{L})$ dos extratos brutos das folhas e raízes ou das suas frações foi aplicada na superfície interna da orelha esquerda $(2,5 \mathrm{mg} /$ orelha). A orelha direita recebeu apenas o solvente das amostras $(20 \mu \mathrm{L})$. Após 6 h, os animais foram sacrificados e as orelhas seccionadas em discos circulares de $6 \mathrm{~mm}$ de diâmetro. Estas secções foram pesadas em balança analítica. Utilizou-se a indometacina como controle positivo. Os resultados foram expressos como média \pm erro padrão da média (epm) e analisados utilizando teste de Student para comparação de duas médias, ou análise de variância (ANOVA) para múltiplas comparações, sendo utilizado $\mathrm{P}<0,05$ como nível de significância.

\section{RESULTADOS E DISCUSSÃO}

O estudo fitoquímico das folhas de G. pohliana resultou no isolamento da mistura de esteroides $\beta$-sitosterol, estigmasterol e campesterol, dos ácidos ursólico $(\mathbf{1})^{7}$ e oleanólico $(\mathbf{2}),{ }^{7}$ do ácido pomólico (3) e loliolida (4) como mistura, do 3-O- $\beta$-glicopiranosil$24 \alpha$-etil-colesta-5-enol (daucosterol), ${ }^{8}$ da mistura dos triperpenos ácidos rotúndico (5), 3 $3,6 \beta, 19 \alpha, 23$-tetra-hidroxiurs-12-en-28-oico (6) e clétrico (7), do ácido 5-O-cafeoilquínico (8) e do secoiridoide secoxiloganina (9). As estruturas das substâncias isoladas foram elucidadas com base na análise dos dados espectroscópicos $\left(\mathrm{RMN}{ }^{1} \mathrm{H}\right.$ e ${ }^{13} \mathrm{C}$, DEPT, COSY, HMQC, HMBC e NOESY) e por comparação com os dados disponíveis na literatura.

A mistura das substâncias $\mathbf{3}$ e $\mathbf{4}$ foi obtida da fração clorofórmica como cristais brancos. O espectro de RMN ${ }^{1} \mathrm{H}$ apresentou, dentre outros, dois sinais característicos de hidrogênios vinílicos em $\delta_{\mathrm{H}} 5,27$ $(t, J=3,6 \mathrm{~Hz})$ e $\delta_{\mathrm{H}} 5,74(s)$, integrados em diferentes proporções, que indicaram a presença de duas substâncias. A análise dos dados de RMN ${ }^{1} \mathrm{H} \mathrm{e}^{13} \mathrm{C}$ permitiu definir para a substância majoritária $3 \mathrm{o}$ esqueleto triterpênico da série ursano, principalmente pelos sinais
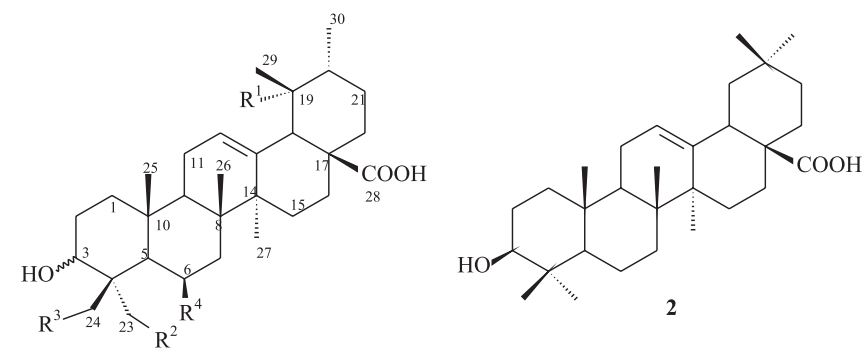

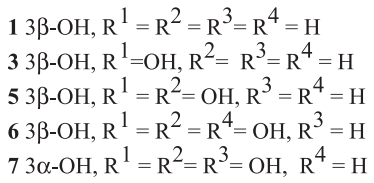
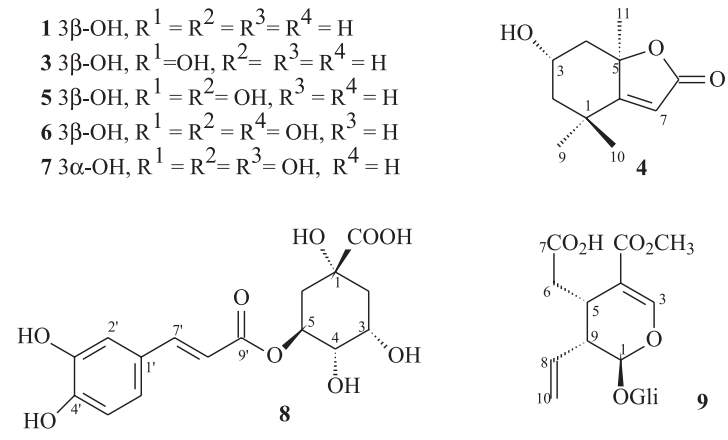

em $\delta_{\mathrm{C}} 129,5$ e 140,0, relacionados aos carbonos olefínicos C-12 e C-13. Foram ainda observados sinais em $\delta_{C} 182,3$, correspondente ao carbono da carboxila de grupo ácido, e em $\delta_{\mathrm{C}} 79,8$, relativo a carbono hidroximetínico. A presença de sinal em $\delta_{\mathrm{C}} 73,6$ referente a carbinólico terciário e o efeito de desproteção observado nos deslocamentos químicos de C-12 $\left(\delta_{\mathrm{C}} 129,5\right)$ e C-20 $\left(\delta_{\mathrm{C}} 43,1\right)$, quando comparados com os de $1,{ }^{8}$ além do singleto observado em $\delta_{\mathrm{H}} 2,49$ no espectro de $\mathrm{RMN}{ }^{1} \mathrm{H}$ e atribuído ao $\mathrm{H}-18$, possibilitaram inferir um grupo hidroxila na posição C-19. A comparação dos dados espectroscópicos com os da literatura ${ }^{7,9}$ permitiu identificar a substância 3 como o ácido pomólico.

Nos espectros de $\mathrm{RMN}{ }^{1} \mathrm{H}$ e ${ }^{13} \mathrm{C}$ da mistura $\mathbf{3}$ e $\mathbf{4}$ ainda foram observados sinais adicionais, entre os quais, em $\delta_{\mathrm{C}} 185,7 ; 174,1$; 113,3 e 67,3, que indicaram para a substância 4 uma unidade lactônica $\alpha, \beta$-insaturada. A comparação dos dados espectroscópicos com os da literatura ${ }^{10}$ permitiu identificar a substância 4 como sendo o isoprenoide loliolida.

Os espectros de $\mathrm{RMN}{ }^{1} \mathrm{He}^{13} \mathrm{C}$ da mistura das substâncias 5, 6 e 7 apresentaram sinais característicos de triterpenos da série ursano semelhantes ao ácido pomólico (3) com grupos hidroxila adicionais em seus esqueletos. A análise dos dados de $\mathrm{RMN}{ }^{1} \mathrm{H}$ permitiu identificar três singletos largos em $\delta_{\mathrm{H}} 5,61 ; 5,72$ e 5,63 correspondentes aos hidrogênios vinílicos (H-12), e de três singletos em $\delta_{\mathrm{H}} 3,11 ; 3,12$ e 3,07 correspondentes a hidrogênios metínicos $(\mathrm{H}-18)$ para 5, 6, e 7, respectivamente. Foram ainda observados sinais de duplo dupletos em $\delta_{\mathrm{H}} 4,20(J=11,4$ e 4,2 Hz, H-3) e 4,30 ( $J=11,4$ e 4,2 Hz, H-3), característicos de hidrogênios carbinólicos em triterpenos $3 \beta-\mathrm{OH}$ e de um singleto largo em $\delta_{\mathrm{H}} 4,74(\mathrm{H}-3)$, característico de hidrogênios carbinólicos $3 \alpha-\mathrm{OH}$. Além destes, foram observados sinais adicionais na região característica de hidrogênios carbinólicos. $\mathrm{O}$ sinal em $\delta_{H} 5,08$, referente a hidrogênio hidroximetínico, foi atribuido ao H-6, evidenciando a presença do grupo $6 \beta-\mathrm{OH}$ em 6. Os pares de hidrogênios hidroximetilênicos em $\delta_{\mathrm{H}} 4,07 / 4,43(d, J=10,2 \mathrm{~Hz})$; $4,37 / 4,58(d, J=10,8 \mathrm{~Hz})$ e 4,02/4,20 $(d, J=10,5 \mathrm{~Hz})$ indicaram a presença dos grupos $\mathrm{OH}$ nas posições $\mathrm{C}-23$ para 6 e C-23 e C-24 para 7, respectivamente. $\mathrm{O}$ espectro de $\mathrm{RMN}{ }^{13} \mathrm{C}$ apresentou sinais relacionados aos carbonos olefínicos C-12 e C-13 em $\delta_{\mathrm{C}} 129,3$ e 140,0 (5); 129,7 e 139,3 (6) e 129,4 e 139,9 (7), os sinais referentes a carbonos carbinólicos atribuídos para 5 : $\delta_{\mathrm{C}} 73,8 ; 73,6$ e 67,3 ; para $\mathbf{6}$ : $\delta 73,6 ; 68,2 ; 73,6$ e 67,5 ; e para $7: \delta 70,8 ; 73,6 ; 68,9$ e 64,8 e o sinal 
em $\delta_{\mathrm{C}} 182,3$ correspondente aos carbonos carboxilícos C-28 para as três substâncias. A comparação dos dados espectroscópicos com os dados da literatura permitiu identificar as substância 5, 6 e 7 como o ácido rotúndico, ${ }^{11}$ o ácido $3 \beta, 6 \beta, 19 \alpha, 23$-tetra-hidroxiurs-12-em28-oico ${ }^{4,12}$ e o ácido clétrico, respectivamente. ${ }^{13} \mathrm{O}$ ácido rotúndico já havia sido isolado nas espécies G. platypoda,$^{11}$ G. angelica ${ }^{14}$ e $G$. grazielae $^{4}$ e o triterpeno 6 já havia sido encontrado no caule de $G$. grazielae. ${ }^{4} \mathrm{O}$ ácido clétrico está sendo descrito pela primeira vez no gênero, mas na família Rubiaceae já foi relatada sua presença em Morinda citrifolia (Noni), ${ }^{15}$ Psychotria correae ${ }^{16}$ e Mussaenda pubescens, ${ }^{13}$ espécies consideradas medicinais.

A substância 8 foi isolada da fração AcOEt como sólido amorfo e caracterizada como ácido 5-O-cafeoilquínico, através da comparação dos dados espectroscópicos de RMN com os descritos na literatura. ${ }^{17}$ No espectro de $\mathrm{RMN}{ }^{1} \mathrm{H}$ de $\mathbf{8}$ foram observados sinais característicos de uma unidade cafeoíla, devido à presença de dois dupletos em $\delta_{\mathrm{H}}$ 7,58 e 6,32 referentes a hidrogênios olefínicos em relação de acoplamento trans $(d, J=15,9 \mathrm{~Hz})$, além de sinais correspondentes a aromático 1,3,4-trissubstituído. A posição do grupo cafeoíla em C-5 do esqueleto ácido quínico foi inferida com base no efeito de desproteção observado para $\mathrm{H}-5\left(\delta_{\mathrm{H}} 5,33, d d d, J=10,8 ; 10,8\right.$ e $\left.4,5 \mathrm{~Hz}\right)$ quando comparado ao H-3 $\left(\delta_{\mathrm{H}} 3,87, d d, J=9,9\right.$ e $\left.3,3 \mathrm{~Hz}\right)$, aliado às constantes de acoplamento características dos mesmos. Esta posição também foi confirmada pelo mapa de contornos $\mathrm{HMBC}$ através da correlação de H-5 com C-9' $\left(\delta_{\mathrm{C}} 168,8\right)$.

A substância 9 foi isolada da fração hidrometanólica como um sólido amorfo e caracterizada como o secoiridoide secoxiloganina, através da comparação dos dados espectroscópicos de RMN com os descritos na literatura. ${ }^{18}$ Substâncias desta classe são consideradas marcadores quimitaxonômicos da subfamília Antirheoideae, à qual pertence o gênero Guettard $a,{ }^{19}$ e secoxiloganina também foi verificada em G. platypoda. ${ }^{20}$

Ensaios de atividade anti-inflamatória foram realizados para o extrato bruto das folhas e de suas frações hexânica, clorofórmica, acetato de etila e hidrometanólica, bem como para o extrato bruto das raízes e suas frações acetato de etila e hidrometanólica. Utilizouse o modelo de edema de orelha induzido por óleo de cróton em camundongo. Os resultados mostraram que a administração na dose de 2,5 mg, por via tópica do extrato bruto e frações das folhas, inibiu o edema em $65 \%$ para o extrato bruto, $68 \%$ para a fração hexânica, $38 \%$ para a fração clorofórmica, 55\% para a fração acetato de etila e $46 \%$ para a fração hidrometanólica. O extrato bruto e as frações acetato de etila e hidrometanólica das raízes inibiram o edema em 46, 43 e $22 \%$, respectivamente, com a administração da mesma dosagem.

A atividade anti-inflamatória tópica dos ácidos ursólico, oleanólico, pomólico, rotúndico e quinóvico já é bastante conhecida. ${ }^{21}$ A presença destas substâncias em G. pohliana contribui para a significativa atividade observada no extrato bruto e frações hexânica e clorofórmica provenientes das folhas. A presença dos ácidos rotúndico e quinóvico no gênero vem justificar o uso popular das espécies no tratamento de ferimentos e inflamações.

\section{CONCLUSÃO}

O estudo fitoquímico das folhas de Guettarda pohliana resultou no isolamento de esteroides, dos triterpenos ácidos ursólico, oleanólico, pomólico, rotúndico, 3 $3,6 \beta, 19 \alpha, 23$-tetra-hidroxiurs-12-en-28oico e clétrico, do isoprenoide loliolida, de daucosterol, do ácido 5- $O$ cafeoilquínico e do secoiridoide secoxiloganina. Os extratos brutos das folhas e raízes e frações provenientes destes extratos apresentaram significativa atividade anti-inflamatória para o modelo de edema de orelha. A presença de triterpenos pentacíclicos dos tipos ursano e oleanano, e também de saponinas derivadas do ácido quinóvico, foi verificada em outras espécies do gênero, especialmente nas plantas medicinais do nordeste brasileiro G. angelica e G. platypoda. Esta última também apresentou atividade anti-inflamatória. Em ambas estão presentes os ácidos rotúndico e quinóvico e as saponinas. Os ácidos clétrico, oleanólico e pomólico estão sendo descritos pela primeira vez no gênero.

\section{AGRADECIMENTOS}

Ao CNPq e à CAPES pelas bolsas concedidas.

\section{REFERÊNCIAS}

1. Achille, F.; Motley, T. J.; Lowry, P. P.; Jeremie, J.; Ann. Missouri Bot. Gard. 2006, 93, 103.

2. Capasso, A.; Balderrama, L.; Sivila, S. C.; Tommasi, N. D.; Sorrentino, L.; Pizza, C.; Planta Med. 1998, 64, 348.

3. Agra, M. F.; Freitas, P. F.; Barbosa-Filho, J. M.; Rev. Bras. Farmacogn. 2007, 17, 114; Bhattacharyya, J.; de Almeida, M. Z.; J. Nat. Prod. 1985, $48,148$.

4. Lima, G. S.; Moura, F. S.; Lemos, R. P. L.; Conserva, L. M.; Rev. Bras. Farmacogn. 2009, 19, 284.

5. Oliveira, P. R. N.; Testa, G.; Sena, S. B.; Sarragiotto, M. H.; Souza, M. C.; Costa, W. F.; Santin, S. M. O.; Quim. Nova 2008, 31, 755.

6. van Arman, G. C.; Clinical Pharmacology and Therapeutics 1974, 16, 900.

7. Mahato, S. B.; Kundu, A. P.; Phytochemistry 1994, 37, 1517.

8. Lendl, A.; Werner, I.; Glasl, S.; Kletter, C.; Mucaji, P.; Presser, A.; Reznicek, J.; Taylor, D. W.; Phytochemistry 2005, 66, 2381.

9. Santos, R. P.; Lemos, T. L. G.; Pessoa, O. D. L.; Braz-Filho, R.; Rodrigues-Filho, E.; Viana, F. A.; Silveira, E. R.; J. Braz. Chem. Soc. 2005, 16, 662 .

10. Cogenero, L. S.; Ide, R. M.; Nazari, A. S.; Dias Filho, B. P.; Nakamura, C. V.; Carvalho, J. E.; Foglio, M. A.; Sarragiotto, M. H.; Quim. Nova 2003, 26, 825 .

11. Soares, F. P.; Ronconi, C. A. V.; Cunha, E. V. L.; Barbosa-Filho, J. M.; Silva, M. S.; Braz-Filho, R.; Magn. Reson. Chem. 1998, 36, 608.

12. Ma, X.; Chongren, Y.; Zhang, Y.; Magn. Reson. Chem. 2008, 46, 571.

13. Zhao, W.; Wolfender, J.-L.; Hostettmann, K.; Cheng, K.; Xu, R.; Qin, G.; Phytochemistry 1997, 45, 1073.

14. Sousa, M. P.; Matos, M. E. O.; Machado, M. I. L.; Vencato, I.; Mascarenhas, Y. P.; Braz Filho, R.; Phytochemistry 1984, 23, 2589.

15. Junko, T.; Iked, Y.; Komiyama, K.; Hayashi, M.; Kishida, A.; Ohsaki, A.; Chem. Pharm. Bull. 2007, 55, 343.

16. Achenbach, H.; Lottes, M.; Waibel, R.; Karikas, G. A.; Correa, M. D.; Gupta, M. P.; Phytochemistry 1995, 38, 1537.

17. Santos, A. R.; Barros, M. P.; Santin, S. M. O.; Souza, M. C.; Eberlin, M. N.; Meurer, E. C.; Sarragiotto, M. H.; Quim. Nova 2004, 27, 525; Meira, M.; David, J. M.; David, J. P.; Araújo, S. V.; Regis, T. L.; Giulietti, A. M.; Queiróz, L. P.; Quim. Nova 2008, 31, 751.

18. Boros, C. A.; Stermitz, F. R.; J. Nat. Prod. 1991, 54, 1173.

19. Inouye, H.; Takeda, Y.; Nishimura, H.; Kanomi, A.; Okuda, T.; Puff, C.; Phytochemistry 1988, 27, 2591.

20. Aquino, R.; De Simone, F.; Senatore, F.; Pizza, C.; Pharm. Res. Comm. 1988, 20, 105

21. Tsai, S.-J.; Yin, M.-C.; J. Food Sci. 2008, 73, 174; Schinella, G.; Aquila, S.; Dade, M.; Giner, R.; Recio, M. C.; Spegazzini, P.; Tournier, H.; Rios, J. L.; Planta Med. 2008, 74, 215; Banno, N.; Akihisa, T.; Takuda, H.; Yasukawa, K.; Higashihara, H.; Ukiya, M.; Watanabe, K.; Kimura, Y.; Hasegawa, H.; Nishino, H.; Biosci. Biotechnol. Biochem. 2004, 68, 85; Sosa, S.; Altinier, G.; Politi, M.; Braca, A.; Morelli, I.; Della Loggia, R.; Phytomedicine 2005, 12, 271; Saad, S. F.; Saber, A. A. H.; Scott, P. M.; Bull. Fac. Pharm. 1967, 6, 253. 